\title{
Prevalence of Human Papillomavirus in Women from Mexico City
}

\author{
María Guadalupe López Rivera, ${ }^{1}$ Maria Olivia Medel Flores, ${ }^{2}$ \\ José D'Artagnan Villalba Magdaleno, ${ }^{3,4}$ and Virginia Sánchez Monroy ${ }^{1,5}$ \\ ${ }^{1}$ Laboratorio Multidisciplinario de Investigación, Escuela Militar de Graduados de Sanidad, Universidad del Ejército y Fuerza Aérea, \\ 11620 México, DF, Mexico \\ ${ }^{2}$ Laboratorio de Biomedicina Molecular I, Programa Institucional de Biomedicina Molecular, \\ Escuela Nacional de Medicina y Homeopatía, IPN, 07320 México, DF, Mexico \\ ${ }^{3}$ Universidad del Valle de México, Campus Chapultepec, Avenida Constituyentes 151, Col. San Miguel Chapultepec, \\ 11850 México, DF, Mexico \\ ${ }^{4}$ Escuela Medico Militar, Universidad del Ejército y Fuerza Aérea, 11620 México, DF, Mexico \\ ${ }^{5}$ Centro de Estudios Científicos y Tecnológicos No. 6 "Miguel Othón de Mendizábal”, IPN, Avenida Jardín S/N, Col. Del Gas, \\ Azcapotzalco, 02950 México, DF, Mexico
}

Correspondence should be addressed to Virginia Sánchez Monroy, vickysm17@hotmail.com

Received 7 February 2012; Revised 24 April 2012; Accepted 16 May 2012

Academic Editor: Bryan Larsen

Copyright (C) 2012 María Guadalupe López Rivera et al. This is an open access article distributed under the Creative Commons Attribution License, which permits unrestricted use, distribution, and reproduction in any medium, provided the original work is properly cited.

\begin{abstract}
Introduction. Cervical cancer is the most common cancer among Mexican women. The goal of the present study was to determine the prevalence and distribution of HPV types in women from Mexico City. Methods. Our study was conducted in the Clinica de Especialidades de la Mujer de la Secretaría de la Defensa Nacional, Mexico. Random samples were taken from 929 healthy women requesting a cervical Papanicolaou examination. Detection and genotyping of HPV were performed by multiplex PCR, with the HPV4A ACE Screening kit (Seegene). Results. 85 of nine hundred twenty-nine women (9.1\%) were infected with HPV. Of HPVpositive women, $99 \%$ and $1 \%$ had high- and low-risk HPV genotypes, respectively. The prevalence of the 16 high-risk (HR) HPV types that were screened was 43\%:42\% (18) were HPV positive and 14\% (16) were HPV positive, which includes coinfection. Multiple infections with different viral genotypes were detected in $10 \%$ of the positive cases. Abnormal cervical cytological results were found in only $15.3 \%$ of HPV-positive women, while $84.7 \%$ had normal cytological results. Conclusions. We found a similar prevalence of HPV to previous studies in Mexico. The heterogeneity of the HPV genotype distribution in Mexico is evident in this study, which found a high frequency of HPV HR genotypes, the majority of which were HPV 18.
\end{abstract}

\section{Introduction}

Cervical cancer is the second most common cancer among women worldwide [1] and the most common cancer among women in Mexico [2].

Cervical cancer is caused by oncogenic HPV infection. More than 100 HPV genotypes have been described, of which approximately 40 are responsible for genital infection [3]. HPV is classified as low or high risk based on its association with premalignant and malignant lesions, respectively. Lowrisk HPV types include $6,11,40,42,43,44,54,61,70,72$, and 81 . Of these types, 6 and 11 cause $90 \%$ of the external anogenital wart cases and low-grade changes in cervical cells.
$[4,5]$. The high-risk types include $16,18,31,33,35,45,52$, and 58 , of which 16 and 18 cause approximately $70 \%$ of all invasive cervical cancer cases [6].

Some authors have suggested that geographical differences in HPV distribution may have an impact on the effectiveness of the HPV vaccine in different populations [7].

In 2005, the International Agency for Research on Cancer HPV [8] reported the worldwide distribution of HPV types in women with normal cervical cytology. The most frequent type is HPV 16, followed by HPV 42, 58, 31, 18, 56, 81, 35, 33, and 45. In Mexico, HPV types 16, 18, 31, 33, 39, 45, 53, 58 , and 59 have frequently been found in normal cervical samples [9-15]. 
Regional data on the prevalence and type distribution of HPV are essential for estimating the impact of vaccines on cervical cancer and developing screening programs.

The goal of the present study was to determine the prevalence and distribution of HPV types in women from Mexico City.

\section{Materials and Methods}

2.1. Study Population. This study was conducted in the Clinica de Especialidades de la Mujer de la Secretaría de la Defensa Nacional in Mexico City, Mexico. Random samples were taken from healthy women requesting a cervical Papanicolaou examination. A total of 929 women, aged between 18 and 76 years, were recruited for the analysis. Written informed consent was obtained from each participant. The protocol and informed consent documents were approved by the Human Research Ethical Committee of the Clínica de Especialidades de la Mujer de la Secretaría de la Defensa Nacional.

2.2. Specimen Collection. Two cervical samples were obtained from each patient using a cytobrush for cytological analysis and HPV detection. Cervical smears were used for cytomorphological examination using conventional Papanicolaou (Pap). For HPV detection, cytobrushes with cervical scrapes were placed in phosphate buffered saline (PBS) and stored at $-70^{\circ} \mathrm{C}$ until analysis.

2.3. Cytology. Pap smears were interpreted by the head of the Cytopathology Laboratory Pathology Unit at the Clínica de Especialidades de la Mujer de la Secretaría de la Defensa Nacional. All Pap smears were described with the Bethesda System terminology.

2.4. HPV Detection and Typing. DNA extraction was performed with the DNeasy Blood \& Tissue Kit (Qiagen, Hilden, Germany) according to the manufacturer's protocol. HPV detection and genotyping were performed by Multiplex PCR using the HPV4A ACE Screening kit (Seegene) according to the manufacturer's protocol.The kit is a qualitative, in vitro test for the identification of HPV 16 and 18 and for screening of 16 high-risk HPV (HR) types $26,31,33,35,39,45,51,52$, $53,56,58,59,66,68,73$, and 82 and the low-risk HPV types 6 and 11 .

2.5. Statistical Analysis. The mean age and the frequency of risk factors were calculated. Significant differences between groups with or without HPV were determined by the MannWhitney rank sum test and the Fisher's exact test at $P<0.05$. Statistical analyses were performed using the SigmaStat program, version 2.03 .

\section{Results}

3.1. Characteristics of the Population. The mean age of women in the study was 40.5 years old. The majority of participants reported one lifetime sexual partner (97\%) and

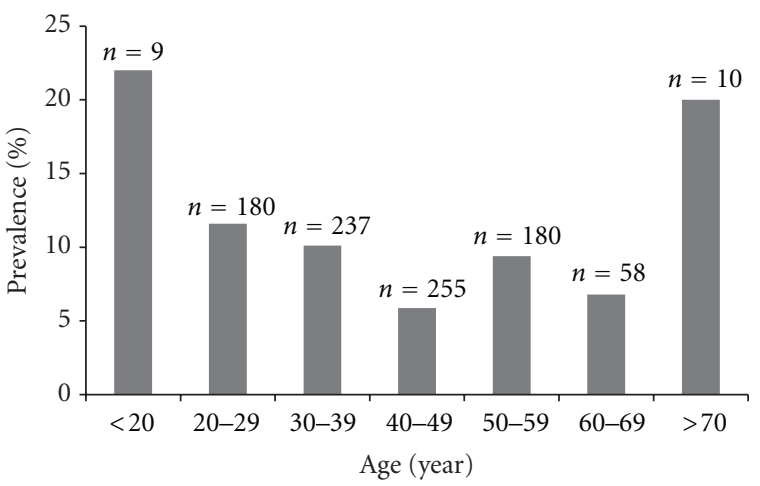

FIGURE 1: Age-specific prevalence of human papillomavirus (HPV) infection among women.

only approximately $3 \%$ of women reported more than one partner in their sexual history. The age of first intercourse was younger than 18 years for approximately $8.6 \%$ of women in the study. Histories of sexually transmitted diseases were rare $(3.9 \%)$. Four percent of the women in the study were current smokers.

3.2. Cytology. Conventional Pap smears identified normal cytological results or inflammation in 916 of 929 samples (98.6\%). Only $13(1.4 \%)$ cases displayed abnormal cytology: $10(1.1 \%)$ had low-grade squamous intraepithelial lesions (LSIL), $2(0.2 \%)$ had atypical squamous cells of undetermined significance (ASC-US), and $1(0.1 \%)$ had squamous cell carcinoma (CC). No high-grade squamous intraepithelial lesions (HSILs) were detected.

3.3. HPV Detection and Genotyping. 85 of 929 women (9.1\%) were infected by HPV. The age-specific prevalence of HPV is summarized in Figure 1, which shows the highest prevalences, $22 \%$ and $20 \%$, in women aged less than 20 years and 70-76 years, respectively. Women aged $40-49$ years old had the lowest prevalence of HPV of $6 \%$.

HPV incidence was significantly higher among women with a history of more than one sexual partner or who were current smokers. There were no differences in the frequency of HPV infection in women who had intercourse younger than 18 years old or those with histories of sexually transmitted diseases (Table 1).

Of the HPV-positive women, 99\% had high-risk HPV genotypes and $1 \%$ had low-risk HPV genotypes. HPV HR was detected in $43 \%$ of women screened in this study: $42 \%$ (18) were HPV positive, and 14\% (16) were HPV positive, which includes those with both infections. Multiple infections with different viral genotypes were detected in $10 \%$ of the positive cases. Abnormal cervical cytology was detected in only $15.3 \%$ of HPV-positive women, and normal cytology was found in the other $84.7 \%$ of those cases, as summarized in Table 2.

\section{Discussion}

In this study, we identified a $9.1 \%$ prevalence of HPV: $84.7 \%$ of women had normal cytology and $15.3 \%$ of 
TABLE 1: Risk factors for HPV infection between HPV-negative and HPV-positive women.

\begin{tabular}{|c|c|c|c|}
\hline Characteristics & HPV-negative $n(\%)$ & HPV-positive $n(\%)$ & $P$ \\
\hline Number of women & $844(90.9)$ & $85(9.1)$ & \\
\hline Mean age (years) & 41.4 & 39.6 & 0.158 \\
\hline Intercourse younger than 18 years old & $72(8.5)$ & $8(9.4)$ & 0.349 \\
\hline History of having more than one sexual partner & $22(2.6)$ & $5(5.8)$ & $<0.001$ \\
\hline Smokers & $31(3.7)$ & $4(4.7)$ & 0.016 \\
\hline History of sexually transmitted diseases & $34(4.0)$ & $2(2.3)$ & 0.157 \\
\hline
\end{tabular}

TABLE 2: Prevalence of HPV genotypes.

\begin{tabular}{|c|c|c|c|c|c|c|}
\hline \multirow{3}{*}{$\begin{array}{l}\text { Cytological } \\
\text { diagnosis }\end{array}$} & \multicolumn{6}{|c|}{ HPV genotypes } \\
\hline & & HPV-18 & HPV-16 & HPV-HR/HPV-18** & HPV-HR/HPV-16*** & $\begin{array}{c}\text { HPV- } 6 \text { and } \\
\text { HPV- } 11\end{array}$ \\
\hline & $n(\%)$ & $n(\%)$ & $n(\%)$ & $n(\%)$ & $n(\%)$ & $n(\%)$ \\
\hline Total $(n=85)$ & $37(43)$ & $30(35)$ & $8(10)$ & $6(7)$ & $3(4)$ & $1(1)$ \\
\hline Normal & $28(33)$ & $30(35)$ & $7(9)$ & $5(6)$ & $2(3)$ & 0 \\
\hline ASC-US & $1(1)$ & 0 & 0 & $1(1)$ & 0 & 0 \\
\hline LSIL & $7(8)$ & 0 & $1(1)$ & 0 & $1(1)$ & $1(1)$ \\
\hline HSIL & 0 & 0 & 0 & 0 & 0 & 0 \\
\hline $\mathrm{CC}$ & $1(1)$ & 0 & 0 & 0 & 0 & 0 \\
\hline
\end{tabular}

ASC-US, atypical squamous cells of undetermined significance; LSIL, low-grade squamous intraepithelial lesions; CC, squamous cell carcinoma; HSIL, highgrade squamous intraepithelial lesions.

*HR (16 high-risk HPV types): 26, 31, 33, 35, 39, 45, 51, 52, 53, 56, 58, 59, 66, 68, 73, and 82.

** Detection of multiple infections for HPV 18 and HR.

*** Detection of multiple infections for HPV 16 and HR.

women had abnormal cytology. The worldwide prevalence of HPV in women with normal cytology has been previously reported [8]. Other HPV studies in Mexico using an open population of women with normal cervical cytology reported an incidence of HPV between 4.8 and $43.6 \%$ [9, 13]. A possible explanation for the difference in the reported HPV prevalences includes variables related to HPV acquisition, such as age, age of first sexual intercourse, number of lifetime sexual partners, socioeconomic status, education level, parity, marital status, number of pregnancies, use of hormonal contraceptives, smoking, and interregional variation $[2,9,15]$.

Similar to previous age-specific studies, both in Mexico and other regions of the world, we found two age groups with higher HPV prevalences, women less than 20 years old and women $70-76$ years old. The younger age group $(<20$ years old) with a higher incidence of HPV infection may be an indicator of sexual transmission, as it coincides with the initiation of sexual activity. The older age group (70-79 years old) of HPV-positive women may have been exposed to high rates of HPV transmission when they were young or may have reactivated latent HPV infections by factors associated with older age [14].

The heterogeneity of HPV genotype distribution in Mexico is evident in this study, with the major frequency of HPV HR at 43\%, followed by HPV 18 at $42 \%$ (including coinfection). The genotype distribution could be explained by the dynamic population in Mexico City.

Similar to previous studies, statistically significant differences in HPV incidence were found among women with a history of more than one sexual partner or who were smokers. No significant differences in HPV frequency were found in women who had intercourse younger than 18 years old or had histories of sexually transmitted diseases. These results could have important implications for future screening procedures to assist the prevention of cervical cancer in Mexico.

The differences in HPV prevalence and distribution identified in this study have a potential impact on the effectiveness of HPV vaccinations, which may be investigated in future studies.

\section{Acknowledgments}

The authors thank the Clinica de Especialidades de la Mujer and the women who provided personal information and biological samples for this study.

\section{References}

[1] J. Ferlay, F. Bray, P. Pisani, and D. M. Parkin, "Globocan 2002 cancer incidence. Mortality and prevalence worldwide," IARC Cancer Base no 5, version 2.0, IARC Press, Lyon, France, 2004.

[2] E. C. Lazcano-Ponce, R. A. Rascón-Pacheco, R. LozanoAscencio, and H. E. Velasco-Mondragón, "Mortality from cervical carcinoma in Mexico: impact of screening, 19801990," Acta Cytologica, vol. 40, no. 3, pp. 506-512, 1996.

[3] E. M. De Villiers, C. Fauquet, T. R. Broker, H. U. Bernard, and H. Zur Hausen, "Classification of papillomaviruses," Virology, vol. 324, no. 1, pp. 17-27, 2004. 
[4] M. Stanley, "Prophylactic HPV vaccines: prospects for eliminating ano-genital cancer," British Journal of Cancer, vol. 96, no. 9, pp. 1320-1323, 2007.

[5] N. Muñoz, F. X. Bosch, S. De Sanjosé et al., "Epidemiologic classification of human papillomavirus types associated with cervical cancer," New England Journal of Medicine, vol. 348, no. 6, pp. 518-527, 2003.

[6] J. S. Smith, L. Lindsay, B. Hoots et al., "Human papillomavirus type distribution in invasive cervical cancer and high-grade cervical lesions: a meta-analysis update," International Journal of Cancer, vol. 121, no. 3, pp. 621-632, 2007.

[7] I. N. Mammas, D. Vageli, and D. A. Spandidos, "Geographic variations of human papilloma virus infection and their possible impact on the effectiveness of the vaccination programme," Oncology Reports, vol. 20, no. 1, pp. 141-145, 2008.

[8] G. M. Clifford, S. Gallus, R. Herrero et al., "Worldwide distribution of human papillomavirus types in cytologically normal women in the International Agency for Research on Cancer HPV prevalence surveys: a pooled analysis," The Lancet, vol. 366, no. 9490, pp. 991-998, 2005.

[9] A. Orozco-Colín, A. Carrillo-García, A. Méndez-Tenorio et al., "Geographical variation in human papillomavirus prevalence in Mexican women with normal cytology," International Journal of Infectious Diseases, vol. 14, no. 12, pp. e1082-e1087, 2010.

[10] B. Illades-Aguiar, L. D. C. Alarcón-Romero, V. Antonio-Véjar et al., "Prevalence and distribution of human papillomavirus types in cervical cancer, squamous intraepithelial lesions, and with no intraepithelial lesions in women from Southern Mexico," Gynecologic Oncology, vol. 117, no. 2, pp. 291-296, 2010.

[11] N. Velázquez-Márquez, M. A. Paredes-Tello, H. Pérez-Terrón, G. Santos-López, J. Reyes-Leyva, and V. Vallejo-Ruiz, "Prevalence of human papillomavirus genotypes in women from a rural region of Puebla, Mexico," International Journal of Infectious Diseases, vol. 13, no. 6, pp. 690-695, 2009.

[12] B. Illades-Aguiar, E. M. Cortés-Malagón, V. Antonio-Véjar et al., "Cervical carcinoma in Southern Mexico: human papillomavirus and cofactors," Cancer Detection and Prevention, vol. 32, no. 4, pp. 300-307, 2009.

[13] L. F. Sánchez-Anguiano, C. Alvarado-Esquivel, M. A. ReyesRomero, and M. Carrera-Rodríguez, "Human papillomavirus infections in women seeking cervical Papanicolaou cytology of Durango, Mexico: prevalence and genotypes," BMC Infectious Diseases, vol. 6, article 27, 2006.

[14] E. Lazcano, R. Herrero, N. Muñoz, A. Cruz, K. V. Shah, and P. Alonso, "Epidemiology of HPV infection among mexican women with normal cervical cytology," International Journal Cancer, vol. 91, no. 3, pp. 412-420, 2001.

[15] M. Torroella-Kouri, S. Morsberger, A. Carrillo et al., "HPV prevalence among mexican women with neoplastic and normal cervixes," Gynecologic Oncology, vol. 70, no. 1, pp. 115120, 1998. 


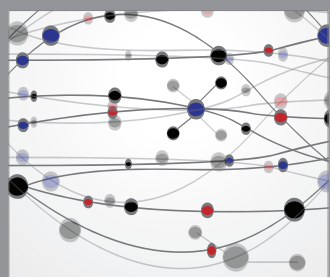

The Scientific World Journal
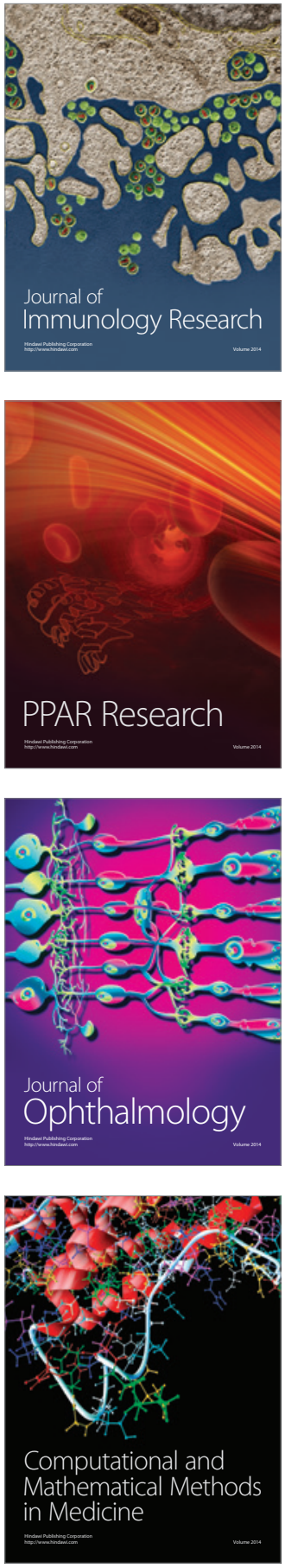

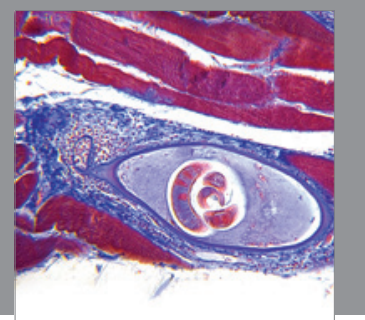

Gastroenterology

Research and Practice
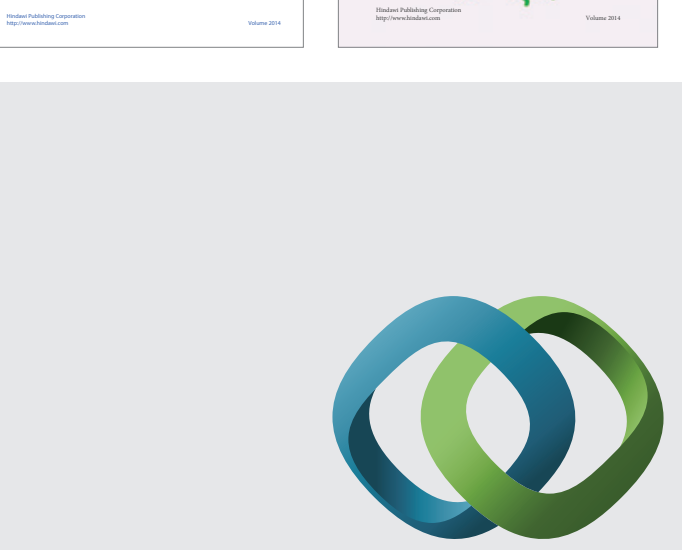

\section{Hindawi}

Submit your manuscripts at

http://www.hindawi.com
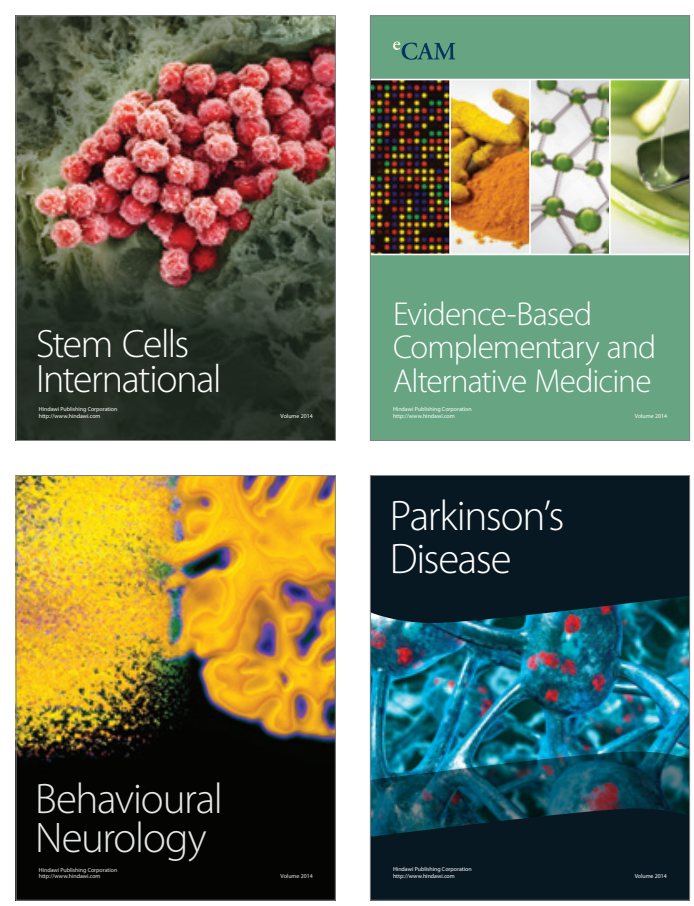

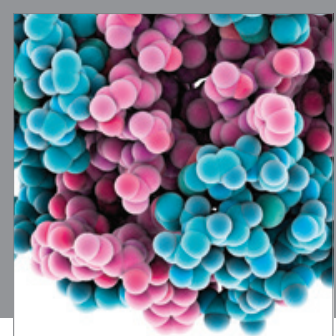

Journal of
Diabetes Research

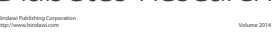

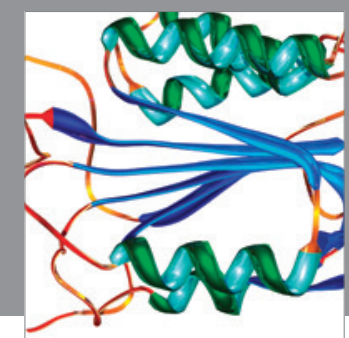

Disease Markers
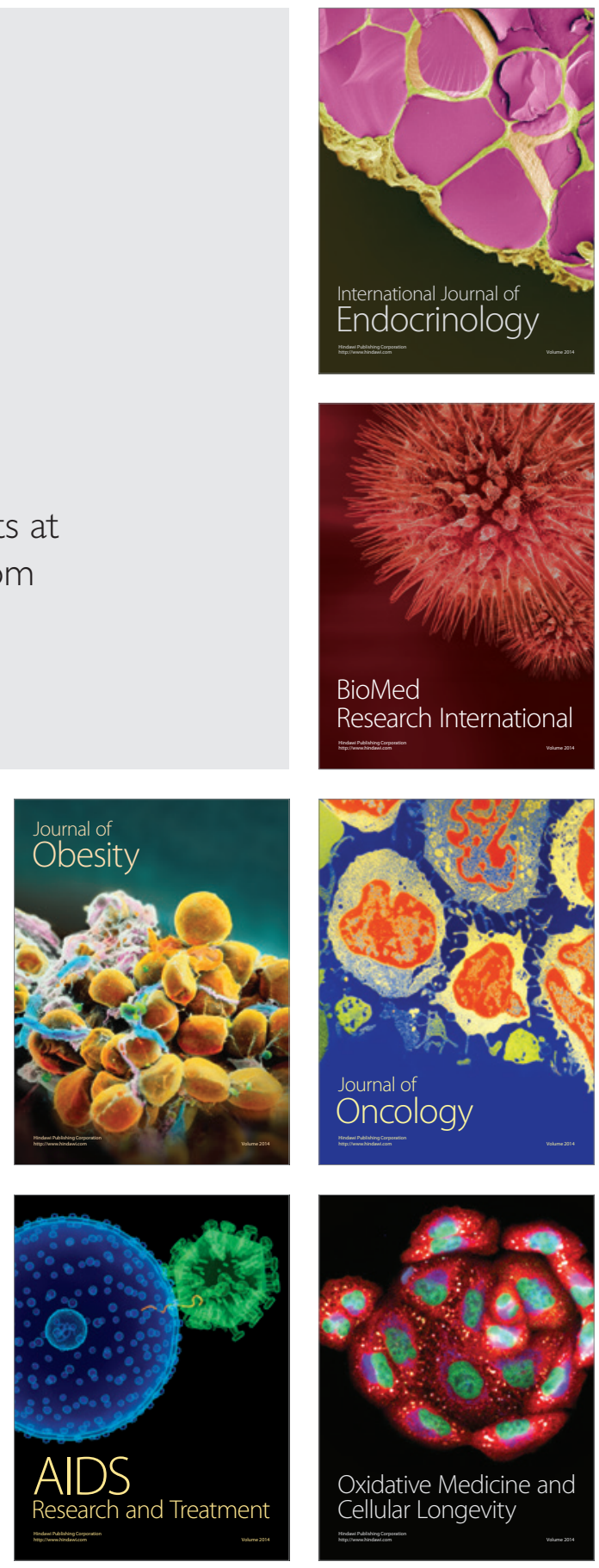